\title{
La implementación del Cloud computing y Big data como herramienta para incrementar la productividad de las Empresas en el Ecuador
}

\section{The implementation of Cloud computing and Big data as a tool to increase the productivity of companies in Ecuador}

\author{
Ing. Washington Geovanny Flores Moreno ${ }^{1}$ \\ geovanny.flores@hotmail.com \\ Ing. Claudia Margarita Avilés Ramírez ${ }^{1}$ \\ margaritaaviles81@hotmail.com
}

Recibido: 1/12/2017, Aceptado: 1/02/2018

\begin{abstract}
RESUMEN
Actualmente el cloud computing, representa un poderoso aliado tecnológico para muchas organizaciones; Todos los usuarios informáticos actualmente tienen acceso es este tipo de servicios. Este servicio está apoyado en la utilización externa de sistemas informáticos y puede ofrecer infinidad funciones a través del Internet, reduciendo enormemente la utilización de dispositivos de almacenamiento físicos y reemplazarlos por virtuales. Esto sumado a un nuevo concepto en el manejo de los datos, un término llamado "BIG DATA" ha llevado a las empresas a un cambio de lo cuantitativo a lo cualitativo, inicialmente se aplicaba este término en la astronomía y genética, pero en la actualidad esto se está llevando hacia todos los ámbitos de la actividad del ser humano. El objetivo general de este trabajo es explicar cómo las empresas del Ecuador a través de la implementación y utilización del cloud computing y el Big Data pueden incrementar su productividad.
\end{abstract}

Palabras clave: Cloud computing, Big data, productividad, servicio, clientes

\section{ABSTRACT}

Currently, cloud computing represents a powerful technological ally for many organizations. All computer users currently have access to this type of services. This service is supported by the external use of computer systems and can offer infinite functions through the Internet, greatly reducing the use of physical storage devices and replacing them with virtual ones. This added to a new concept in data management, a term called "BIG DATA", which has led companies to a change from

\footnotetext{
${ }^{1}$ Universidad Tecnológica Empresarial de Guayaquil. Ecuador
} 


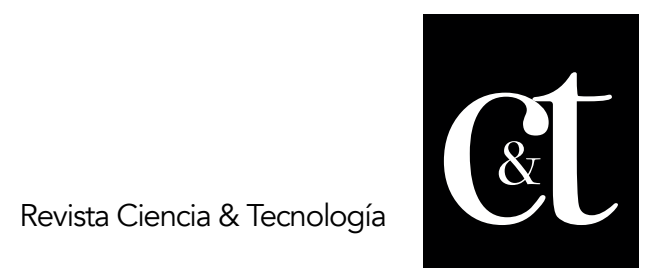

No. 18, 30 de abril de 2018

ISSN impreso: 1390 - 6321

quantitative to qualitative, initially applied this term in astronomy and genetics, but today this is taking place in all areas of human activity. The general objective of this paper is to explain how companies in Ecuador through the implementation and use of cloud computing and Big Data can increase their productivity.

Keywords: Cloud computing, Big data, productivity, service, customers

\section{Introducción}

Debido a la gran reputación que está asumiendo esta tecnología actualmente. Cualquier persona con o sin conocimientos tecnológicos, se debe haber preguntado ¿cómo se almacena toda la información que se genera en el mundo?, así por ejemplo en Facebook, Twitter, Instagram o como Google es capaz de manejar todas las transacciones que se hacen a diario. Para responder a esto, se adoptado el termino Cloud Computing que va más allá de una tendencia tecnológica; y se ha convertido en una necesidad empresarial a la que todos están expuestos y por la que todos deben cautivarse.

Según la revista tecnológica Computerworld (2016), el Cloud es una realidad latente. En el 2015, los usuarios finales ya han gastado más de USD. 180 mil millones en servicios Cloud y se pronostica que, en 2018, el mercado global de Equipamiento Cloud alcanzará los USD. 79.10 mil millones. Estas cifras revelan no solo una inversión en crecimiento, sino también en beneficios donde $82 \%$ de las empresas que han migrado a la Nube reportan haber ahorrado dinero en los procesos posteriores.

El termino Big Data sigue generando confusión, a la cual le asignan muchos términos de uso como son el análisis de las redes sociales, análisis de los datos en tiempo real, análisis de grandes volúmenes de datos que son considerados como información "No SQL" o datos no estructurados (López García, 2013).

En el actual mercado competitivo, los líderes empresariales compiten por convertir la información extraída de los datos en resultados significativos. Aquellos de mayor éxito aplican análisis en toda su organización para tomar decisiones más inteligentes, actuar rápidamente y optimizar los resultados. Cualquiera que fuera el enfoque, queda claro que Cloud Computing ${ }^{2}$ y el Big Data ${ }^{3}$ marca un cambio de paradigma en la informática que radica en hacer de la internet un "servicio", no solo una herramienta informativa.

\footnotetext{
${ }^{2}$ El cloud computing consiste en la posibilidad de ofrecer servicios a través de Internet sin preocuparse por poseer la capacidad suficiente para almacenar información en el computador personal.

3 Big Data es un término que describe el gran volumen de datos, tanto estructurados como no estructurados, que inundan los negocios cada día.
} 


\begin{abstract}
Desarrollo
La presente investigación se basa en un método cualitativo, pues el objetivo está más orientado a describir e interpretar que a medir, desde esta perspectiva, la primera parte de este estudio se define como de tipo documental puesto que consistió en realizar una descripción de las tendencias actuales en tecnología de manejo y almacenamiento de los datos con el propósito de apoyar a empresas ecuatorianas y una segunda parte como un estudio de tipo comparativo, puesto que se trata de varios temas realizados con un mismo enfoque para poder compararlos entre sí y que las empresas tengan una percepción de las distintas opciones disponibles en el mercado.
\end{abstract}

\title{
El Big Data y el cloud computing
}

El avance tecnológico que se está sintiendo durante los últimos años en el mundo de la información y de la comunicación que normalmente se lo conoce como Tecnologías de la Información y comunicación TIC, está obligando a las organizaciones a enfrentarse y adaptarse a diferentes retos, pero ocurre que uno de estos desafíos a captado importancia a lo largo de los últimos años el cual consiste saber cómo administrar, buscar y analizar grandes volúmenes de datos. Con esto surge el problema del almacenamiento por lo que se han acuñado dos términos: El Big Data que se refiere a que las empresas manejen de grandes volúmenes de datos y el Cloud Computing que ayuda a almacenar toda esa cantidad de datos. Todos los días se crean 2,5 quintillones ${ }^{4}$ de bytes de datos, tanto que el $90 \%$ de los datos del mundo actual ha sido creado en los últimos dos años solamente. Estos datos provienen de todas partes como las redes sociales y registros de transacciones de compra por mencionar solo dos ejemplos.

Aguilar (2012) en su libro "Big Data y Open Data: el universo digital de datos" menciona que el auge de los medios sociales, especialmente redes sociales, unido a la prensa digital, fotografías, audio, video ha llevado a las empresas a crear o alquilar espacios de almacenamiento, así como Facebook, y Google, que no paran de crear centros de datos para atender los millones de usuarios. El tamaño para determinar si un conjunto de datos se considera como Big Data no está completamente definido estos datos van desde los 30 o 50 Terabytes a varios Petabytes ${ }^{5}$.

Viñals (2012b) afirma: "No solo es cuestión de volumen, sino también de la velocidad con la que se generan los datos ya estamos en el mundo de la IOT o Internet de las cosas donde todo estará sensorizado". Barranco Fragoso (2012) manifiesta que "Actualmente existe una amplia variedad de tipos de datos que se pueden analizar, clasificarlos de una manera correcta ayuda a entender mejor su representación."

\footnotetext{
${ }^{4} 1$ quintillón $=1030=1,000,000,000,000,000,000,000,000,000,000$ bytes

${ }^{5}$ Petabyte es una unidad de almacenamiento cuyo símbolo es PB equivale a 1015 bytes $=1000000$ 000000000 de bytes.
} 


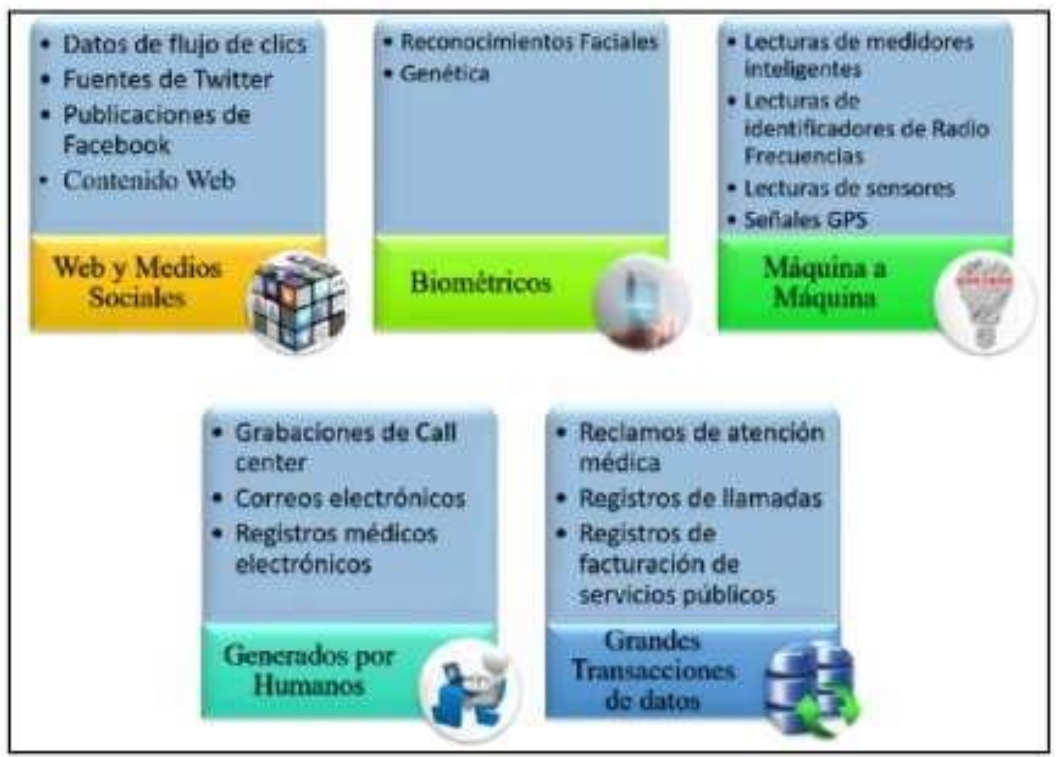

Figura 1. Tipos de Datos del Big Data Fuente: IBM Developer works Elaboración: Los Autores

Las ventajas de utilizar el cloud computing son variadas, por ejemplo: Bajo costo, Seguras, rápidas y el acceso a toda la información cuando quieras y donde quieras. Existen modelos de servicios que las empresas ven muy atractivos, como: Software como Servicio (SAAS), Plataforma como servicio (PAAS), Infraestructura como servicio (IAAS), que solucionan muchos de los problemas que una empresa puede tener.

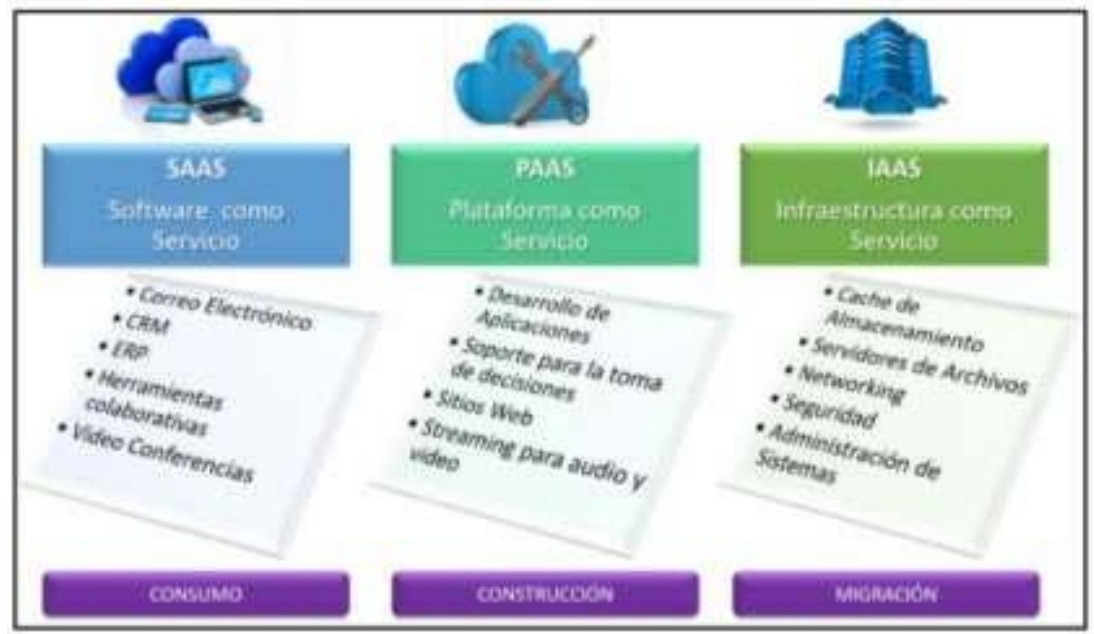

Figura 2. Servicios en la Nube

Fuente: IBM Cloud

Elaboración: Los Autores 
La fortaleza de Cloud Computing o Computación en la nube radica en la capacidad de procesamiento y de almacenamiento de datos, la movilidad y aplicabilidad de la misma (Proaño, 2011).

Consejos antes de confiar los datos de su empresa a un proveedor externo Harry Lewis ya planteaba estas preguntas en el informe especial de BusinessWeek del año 2008, en su artículo "Las nueve preguntas que debe hacerse antes de confiar los datos de su empresa o sus tareas de computación a un proveedor externo" (Aguilar, 2012).

A continuación, se describen algunas de las muchas preguntas que a la hora de contratar un servicio en la nube se deben de tener en cuenta:

- ¿Quién puede ver los datos?

- ¿Cómo se garantiza la privacidad?

- ¿Qué pasa si no se paga la factura mensual o anual?

- ¿Hace la nube copia de seguridad de sus datos?

- ¿Existe un contrato de garantía?

- ¿Cuál es el control de acceso?

- ¿Cuál será la estrategia de salida de la nube?

- ¿Cómo se recuperan datos almacenados?

La adquisición de servicios y soluciones tecnológicas a múltiples proveedores puede suponer que los datos queden expuestos fuera del control de la organización. La seguridad al cien por ciento no existe, para esto se requiere priorizar y controlar la información verdaderamente sensible, si se produce una pérdida de datos $y$, especialmente, si la compañía ha de hacer frente a una sanción de la Agencia de Protección de Datos del país correspondiente, los daños serán difíciles de calcular (Aguilar, 2012).

Los proveedores de cloud computing han evolucionado mucho en materia de seguridad y ofrecen una protección de la privacidad superior a la de cualquier empresa particular, sin embargo, se requiere el control y la certificación de los datos. Los problemas internos son las causas más frecuentes de vulneración de la seguridad, como: Fallos en el sistema o fallos técnicos, empleados negligentes o incompetentes, fallos en los procesos comerciales, delitos cibernéticos, Empleados malicioso.

La constante evolución del comercio móvil junto al incremento en la adopción de Smartphones provoca que cada vez las empresas en el Ecuador desarrollen aplicaciones móviles (App) o páginas web como un nuevo formato de comercialización virtual (Goyes Jiménez, 2017).

Muchas de estas empresas en especial las medianas solo cuentan con el sistema de facturación y en el mejor de los casos con el ERP5. ¿Pero qué tan beneficioso les resultaría implementar soluciones de Big Data y cloud computing?, para responder esta pregunta de debería comprender los siguientes temas.

La visión de $360^{\circ}$ del cliente

Conocer totalmente las necesidades del cliente, diseñar una nueva estrategia de fidelización y extender una red interna de información son algunas de las cosas que una empresa debe considerar y mejorar la visión de 360 del cliente, esto es, 
desplegar un nuevo modelo de gestión enfocado a descubrir una a una las necesidades y preferencias del cliente y también del conjunto de herramientas tecnológicas capaces de obtener, difundir y gestionar correctamente esa información (Cortés, 2012).

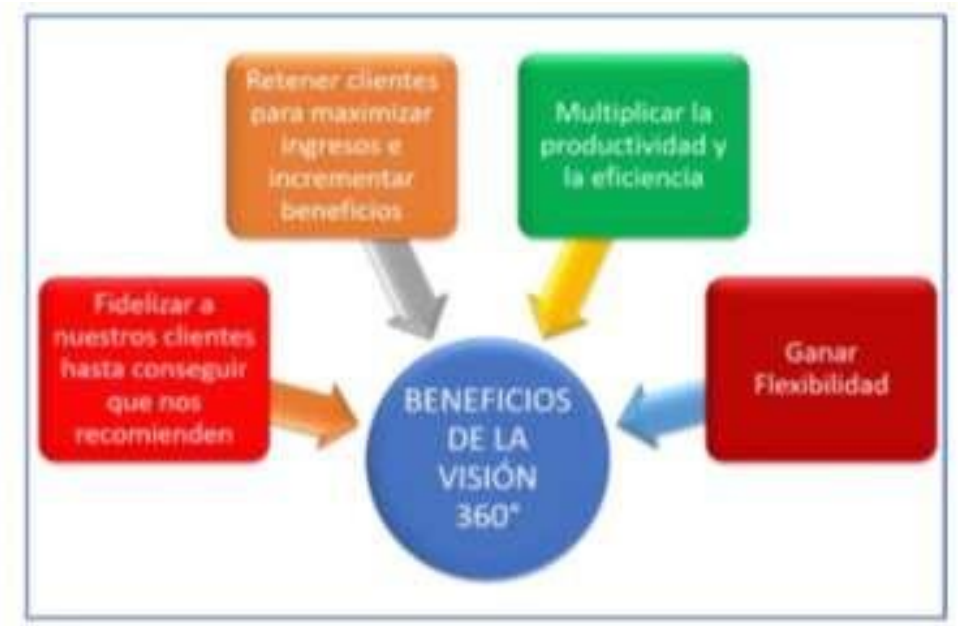

Figura 3. Beneficios de la visión $\mathbf{3 6 0}$

Fuente: Alicia Business Services

Elaboración: Los Autores.

Para reconocer las nuevas oportunidades, es necesario pensar más allá de las fuentes tradicionales de datos, las empresas cuentan con muchas fuentes de datos, como son las redes sociales por ejemplo en donde pueden hacer uso de los comentarios de los usuarios para obtener información valiosa sobre gustos y preferencias, opiniones y quejas y diseñar una estrategia que les permita aprovechar esta información y llegar a todos esos potenciales clientes.

Satisfacer las necesidades de los clientes implica ampliar la visión existente del cliente incorporando fuentes adicionales de información sean estas internas o externas y obtener un mayor entendimiento del sentimiento del cliente, incrementando su satisfacción y conociendo que acciones significativas son necesarias para llegar a ellos proporcionando la información adecuada a la gente adecuada mediante técnicas como Cross-Selling ${ }^{6}$ y Up-Selling7 (Cava, 2004).

\footnotetext{
${ }^{6}$ La técnica del Cross-Selling o venta cruzada implica en mostrar o informar al comprador de otros productos relacionados o que complementan al que quiere comprar.

${ }^{7}$ La técnica del Up-Selling implica inducir al comprador a que elija un producto más caro que el que está comprando.
} 


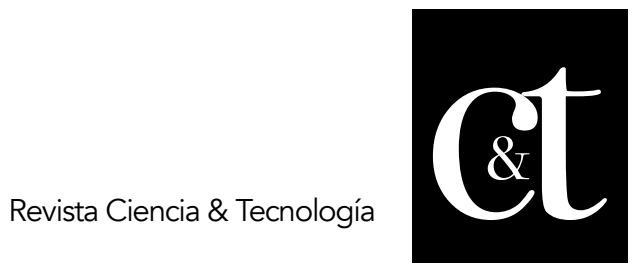

No. 18, 30 de abril de 2018

ISSN impreso: 1390 - 6321

\section{El cloud computing como motor de cambio}

El cloud está aquí y ha venido para quedarse. Lo que veníamos intuyendo en los últimos años se ve confirmado con la aparición diaria de nuevos servicios en la nube o los datos de estudios, como los de CISCO, que nos dicen que dentro de un par de años todo el tráfico de la red tendrá su origen en el cloud computing (Viñals, 2012a).

Ahora bien, si una empresa se limita a adoptar el modelo cloud computing solo para ahorrar dinero, es muy probable que se equivoque de estrategia. La ventaja diferencial del cloud computing es que permite hacer realidad nuevos modelos de negocio.

Pensemos, por ejemplo, que vale lo mismo alquilar una máquina 1.000 horas que 1.000 máquinas una hora. Esto abre un gran abanico de posibilidades a las empresas para probar y ensayar conceptos o procesos. La misma oportunidad se extiende a los emprendedores y las empresas más pequeñas, liberadas de la necesidad de realizar grandes inversiones de capital gracias al modelo de pago por uso descrito (Viñals, 2012a).

Empresas ecuatorianas se proyectan a la computación en la nube

Según el artículo publicado originalmente por la Revista Líderes en el cual Sebastián Pérez, gerente en Ecuador de Nubis Partners, una firma que es socia estratégica de Google señala que. "En Ecuador estamos atrasados en el tema, recién estamos entrando con herramientas básicas como Google Apps. Pero las empresas se están dando cuenta de que pasar a la nube implica un cambio de estrategia de toda la organización" (Revista Líderes, 2012). Las empresas que trabajan utilizando los servicios de la nube, se olvidan de comprar hardware, de mejorar la capacidad de procesamiento ya que toda esta infraestructura ya está contenida en la nube y esto involucra es un cambio de estrategia, es todo un nuevo plan de acción que la empresa debe adoptar.

La velocidad de conexión ha ido en aumentos en los últimos años y ha sido un factor importante para que la Nube se convierta en el medio donde todo confluye y de acuerdo con el informe "Estado de Internet" de AKAMAI, entidad internacional especializada en estudios sobre acceso a las Tecnologías de la Información y Comunicación (TIC), reveló el aumento del tráfico de datos móviles en todo el mundo y que en aquella ocasión trató sobre el tercer trimestre de 2013, donde analizó la velocidad promedio de Internet de 120 países o regiones del mundo, determinando que ésta aumentó en un $29 \%$ en comparación al tercer trimestre de 2012 , alcanzando los 3,6 Mbps promedio a nivel global. 


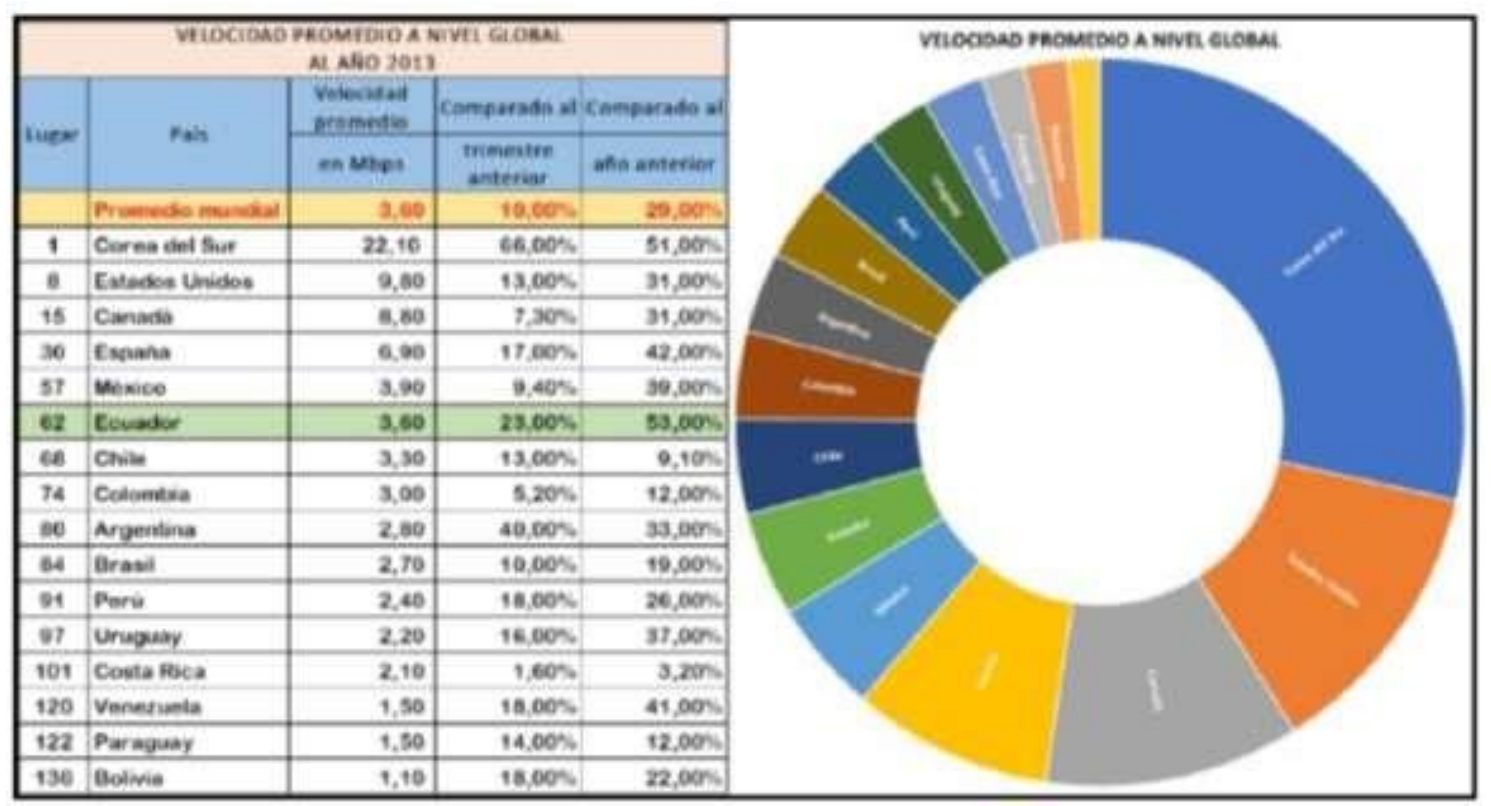

Figura 4. Velocidad promedio de internet a nivel global

Fuente: AKAMAI

Elaboración: Los Autores

Así mismo, gracias a la Gobierno Nacional y el sector privado existe una nueva realidad en nuestro país. Según AKAMAI en el año 2016, Ecuador contó con 40 veces más velocidad de acceso a Internet, el promedio de navegación en el 2006 fue de 128 kbps y en junio de 2016 se contó con 5.1 megabytes por segundo (...) y más de la mitad de la población ecuatoriana usa el Internet (Vallejo, 2017).

La revista Computerworld (2016) en su artículo "Impulsando la competitividad empresarial", el cual pronosticó que el mercado para la Nube pública en 2016 sería de $\$ 205$ mil millones, de acuerdo a Gartner; el valor es superior al 2015 con $\$ 30$ mil millones. Se prevé que su crecimiento se mantenga en una tasa de $16.4 \%$ anual, a medida que más empresas se suman a la ola de la transformación digital.

Se estableció según el estudio denominado "Cloud Confusion", efectuado en el año 2012 por Citrix (2012), una de las principales proveedoras de servicios en la nube a escala global. donde señaló que el $95 \%$ de las personas que utiliza servicios en la nube creía que nunca los ha utilizado lo que demuestra más desconocimiento que falta de uso en ese año. Se está viviendo la revolución del cloud computing como sistema nervioso central de la nueva infraestructura de comunicaciones universal, que resulta más importante que Internet y que el valor de cloud computing es la probabilidad del desarrollo de software que proporcionará grandes posibilidades para dicho segmento y que hasta ahora sólo podían realizar los países más avanzados (Aguilar, 2012). 
Principales proveedores de cloud en el Ecuador

En el Ecuador son pocas las empresas que están desarrollando servicios de Cloud Computing, entre ellas se mencionan a:

- Telconet con su servicio Telconet Cloud Center.

- Microsoft con su servicio Azure.

- Claro con su servicio Claro Cloud.

- IBM con su servicio IBM Cloud.

- Punto Net con su servicio Punto Cloud.

TELCONET. - Es una empresa con operaciones en Ecuador con una trayectoria de más de 21 años en Soluciones de Conectividad, Internet, Centro de Datos y Servicios Gerenciados. Con una sólida plataforma de infraestructura de Fibra Óptica de un altísimo nivel de capilaridad.

MICROSOFT. - Es una de las más grandes empresas desarrolladoras de software del mundo, entre sus productos cuenta con Microsoft Azure que es una creciente colección de servicios en la nube integrados que los desarrolladores y los profesionales de TI utilizan para crear, implementar y administrar aplicaciones a través de su red global de centros de datos.

CLARO. - Tiene la visión de impulsar el desarrollo mediante la conectividad, buscando crear un servicio universal, convencidos de la importancia de reducir la brecha digital con la finalidad mejorar la calidad de vida los ecuatorianos y acercar cada día más a las personas. A través de su red, el $96 \%$ del territorio ecuatoriano poblado tiene acceso a sus servicios de la más avanzada tecnología.

IBM. - Una reconocida empresa multinacional estadounidense de tecnología y consultoría la cual fabrica y comercializa hardware y software para computadoras, y ofrece servicios de infraestructura, alojamiento de Internet y consultoría en una amplia gama de áreas relacionadas con la informática, desde computadoras centrales hasta nanotecnología.

PUNTONET. - PuntoNet provee servicios de telecomunicaciones, entregando soluciones de Internet a clientes personales y corporativos, pudiendo acceder desde su casa, negocio o empresa a todos los servicios y beneficios, su experiencia en el mercado y la excelencia del servicio de PuntoNet los ha llevado a ser reconocidos como uno de los principales proveedores de servicios de Telecomunicaciones del Ecuador.

¿Qué ofrecen estas empresas? Cada empresa tiene diferentes necesidades y distintos requerimientos de TI, para poder satisfacer esas necesidades existen destinitos proveedores de Cloud, los cuales ahora cuentan con incluso más opciones para implementar soluciones híbridas en la Plataforma en la Nube, los clientes se benefician de servicios personalizados y completamente administrados dentro de su mercado local, además de un alto nivel de consistencia técnica en sus ambientes, lo que evita atarse a un solo proveedor e incrementa la flexibilidad y los clientes se pueden enfocar en aumentar la eficiencia, mejorar la productividad de los empleados y reducir los costos operativos. 
Para este trabajo de investigación se ha seleccionado a CLARO y TELCONET para mostrar parte de los servicios que ofrecen.

CLARO ofrece Servidores Virtuales que es un servicio de capacidad de cómputo en la nube que le permite disponer de recursos como procesamiento, almacenamiento, conectividad, entre otros; a través de instancias virtuales de servidores, poniendo a disposición servidores Windows o Linux disponibles en minutos con recursos Cloud.

La empresa garantiza que obtendrá ahorros relacionados con:

- Inversión en equipos.

- Sistemas de monitoreo y seguridad (Antivirus, Firewall).

- Sistemas de respaldo.

- Personal especializado. Las ventajas de utilizar esta plataforma son:

- Disponibilidad del servicio 24/7.

- Aumento capacidad de cómputo de forma inmediata y dinámicamente.

- Operación centralizada y con infraestructura redundante.

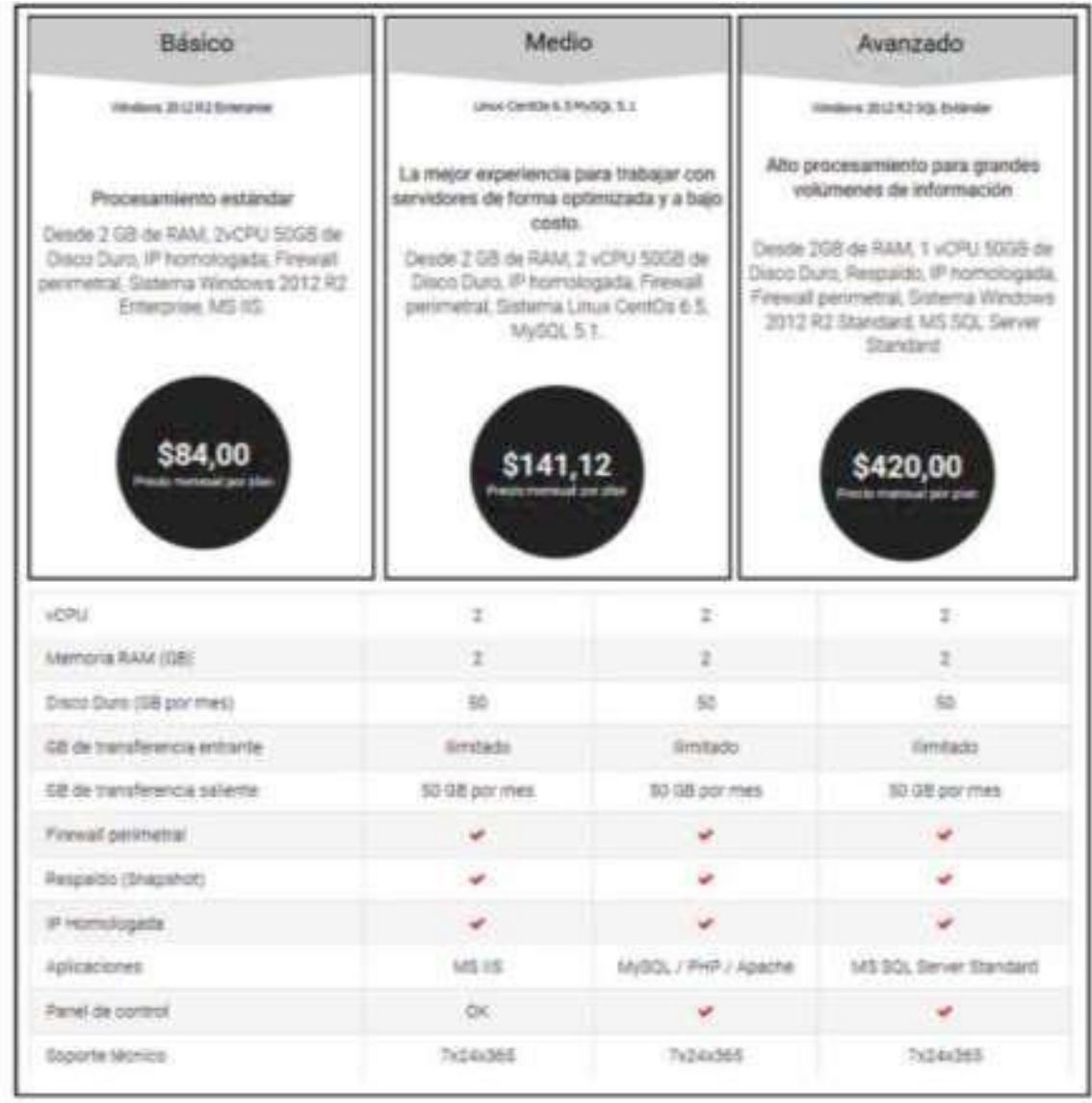

Figura 5. Precios mensuales por alquiler de servidores virtuales ofrecidos por Claro

Fuente: www.claro.com.ec

Elaboración: Los autores 
TELCONET ha diseñado y construido en Ecuador dos Centros de Datos de Categoría Internacional denominados TELCONET CLOUD CENTER I en Guayaquil y TELCONET CLOUD CENTER II en Quito, los cuales se encuentran a la vanguardia de la tecnología y seguridad en infraestructura, permitiendo garantizar los servicios de Housing y Cloud Computing que demandan las empresas.

Estos Centros de Datos están certificados bajo la norma del Uptime Institute en las más altas categorías siendo el centro de datos de Guayaquil TIER ${ }^{8}$ IV y de Quito TIER III, permitiendo formar parte del grupo IDC-G ${ }^{9}$.

Entre los servicios que esta empresa pone a disposición están los siguientes:

- SERVICIOS DE HOUSING (ALOJAMIENTO)

- SERVICIOS DE HOSTING-CLOUD o Respaldo en la Nube o Correo en la nube o Nube Pública

- SERVICIOS ADICIONALES o Instalación, monitoreo y mantenimiento de Hardware Pasivo e Infraestructura Física, monitoreo infraestructura y manos remotas lógicas.

\section{Tabla 1. Valores por servicio BackupNet para Servidores de Telconet}

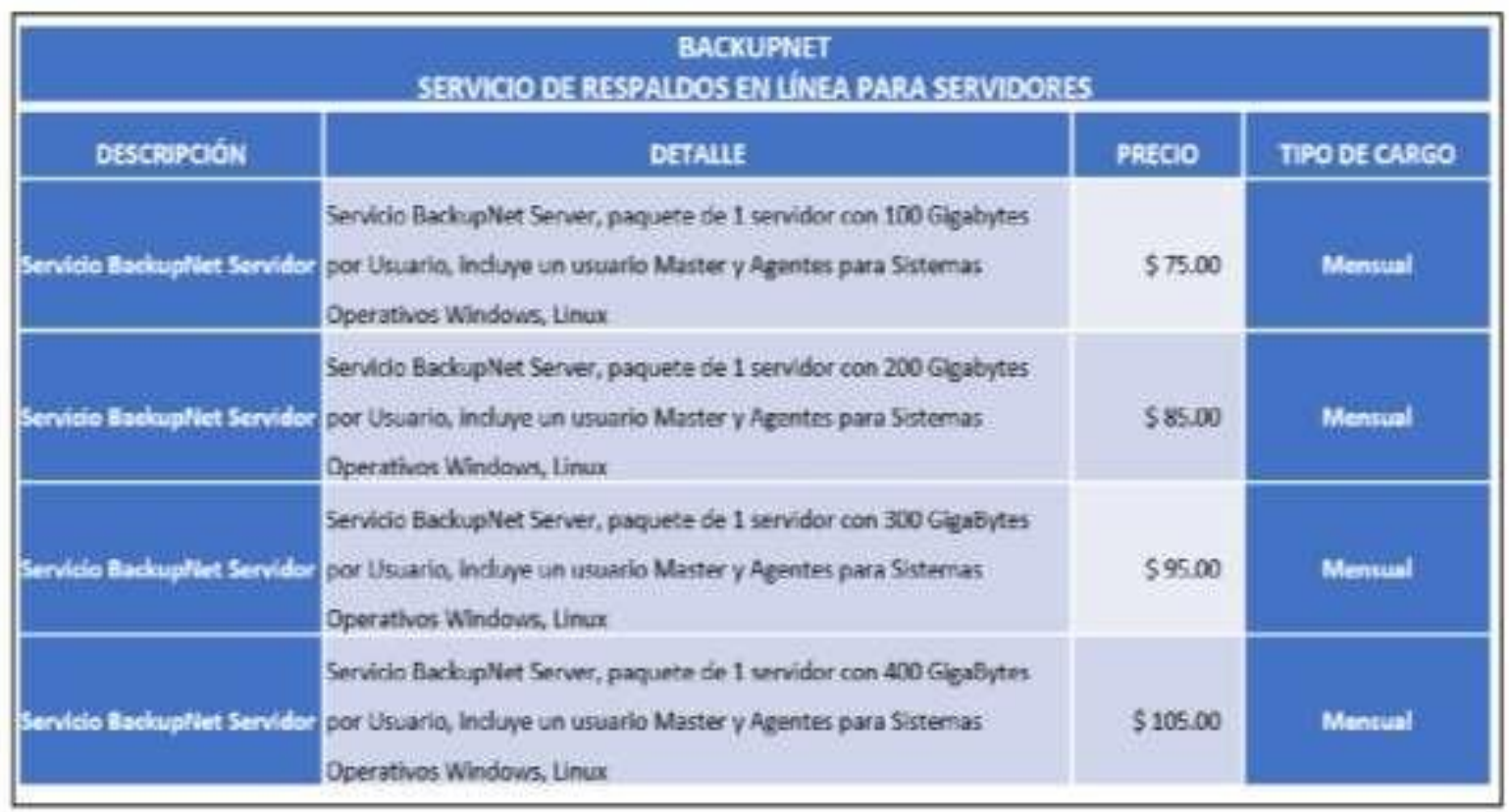

Fuente: Telconet

Elaboración: Los Autores

\footnotetext{
${ }^{8}$ UPTIME INSTITUTE creó el sistema TIER CLASSIFICATION estándar para evaluar de manera efectiva la infraestructura de los centros de datos en términos de los requisitos de una empresa para la disponibilidad de sistemas.

${ }^{9}$ Alianza Internacional de Centro de Datos de Mercados Emergentes
} 
Cabe mencionar que en el ecuador existen cinco centros de datos que cuentan con certificaciones internacionales sobre su infraestructura, esta certificación la otorga el Uptime Institute el cual es una sólida confirmación de que las funciones de administración de TI, ingeniería de centros de datos, finanzas y sostenibilidad están alineadas (Uptime Institute, 2017).

\section{Tabla 2. Certificaciones TIER para Ecuador por Uptime Institute}

\begin{tabular}{|c|c|c|c|}
\hline \multicolumn{4}{|c|}{$\begin{array}{l}\text { CERTIFICACIONESTIER } \\
\text { PARA ECUADOR }\end{array}$} \\
\hline Compañis & Nembre del Centro de Datos & Localización del Centro de Datos & Certificacion TiER \\
\hline \multirow{3}{*}{ 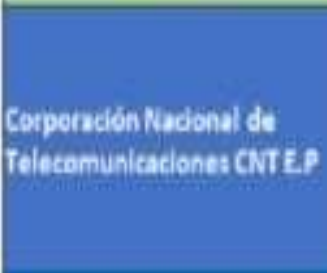 } & \multirow{2}{*}{$\begin{array}{l}\text { Centro de Datos CNTEP - } \\
\text { Ouito }\end{array}$} & \multirow{2}{*}{$\begin{array}{l}\text { Qulto, Pichincha } \\
\text { Ecuador }\end{array}$} & $\begin{array}{l}\text { Ther lill Certification of Constructed } \\
\text { Faclity }\end{array}$ \\
\hline & & & $\begin{array}{l}\text { Ther Illi Certification of Design } \\
\text { Documents }\end{array}$ \\
\hline & $\begin{array}{l}\text { Centro de Datas CNT EP- } \\
\text { Guaraquil }\end{array}$ & $\begin{array}{l}\text { Guayaquil, Guayas } \\
\text { Ecuador }\end{array}$ & $\begin{array}{l}\text { Tler Ill Certification of Design } \\
\text { Documents }\end{array}$ \\
\hline \multirow{2}{*}{ Tolconetsis } & Ielconet Cloud Center I & $\begin{array}{l}\text { Guayaquil, Guayss } \\
\text { Ecuador }\end{array}$ & $\begin{array}{l}\text { Tier IV Centification of Desigy } \\
\text { Documents }\end{array}$ \\
\hline & Ielconet Cloud tenter II & $\begin{array}{l}\text { Quito, Pichincha } \\
\text { Ecuador }\end{array}$ & $\begin{array}{l}\text { Tier III Centification of Design } \\
\text { Documbents }\end{array}$ \\
\hline Concesel 5 A & Claro Data Center Duris & $\begin{array}{l}\text { Duran, Guayas } \\
\text { Eeundor }\end{array}$ & $\begin{array}{l}\text { Tler Ill Certification of Design } \\
\text { Documents }\end{array}$ \\
\hline
\end{tabular}

Fuente: Uptime Institute

Elaboración: Los Autores

\section{Conclusiones}

De este trabajo se han obtenido distintas conclusiones, empezando por averiguar que la tecnología Big Data no solo sirve para obtener grandes cantidades de datos, sino que también sirven para analizar esos enormes volúmenes de datos y conseguir así información y conocimiento.

El uso de Big Data está convirtiendo la gestión de muchas acciones empresariales, permitiendo la apertura de muchas posibilidades para reducir los precios y mejorar los servicios que reciben los clientes o consumidores.

La disponibilidad de grandes bases de datos está también transformando el análisis económico tanto en metodologías como en técnicas específicas.

El incremento de Big Data como parte de actividad mezclado a la actividad empresarial está generando una gran demanda de matemáticos, informáticos y estadísticos con conocimientos de economía.

Se puede determinar ciertos aspectos sensibles como la dependencia en el servicio en los medios de transmisión y la disponibilidad de los mismos, junto con un ideal tiempo de respuesta y con la centralización del proceso de información de una buena parte del aparato económico que estaría sujeta a mayor control y mayor ataque delictivo (hackers, spyware, troyanos). 
El Cloud Computing y el Big Data son tecnologías que están surgiendo poco a poco y que a pesar de que no todas las empresas tienen porque migrar a ella, ha nacido para quedarse y marcar una nueva etapa en el mundo de las Tics y en estos momentos hay organizaciones que poseen grandes cantidades de nuestra información privada y que debe de ser regulada fuertemente.

Por último y no por ello menos importante, a pesar de que hoy en día se diga que estamos en "La era de la Información" en la que se generan enormes volúmenes de datos y lo que actualmente parecen enormes cantidades pronto se convertirían en ínfimas, por ello la tecnología Big Data y Cloud Computing deben y pueden seguir evolucionando, y de este modo aprovechar la gran avalancha de información que se avecina.

\section{Referencias bibliográficas}

Aguilar, L. J. (2012). Computación en la Nube: estrategias de cloud computing en las empresas. México: Alfaomega Grupo Editor S.A.

Barranco Fragoso, R. (18 de 06 de 2012). ¿Qué es Big Data? IBM Developer. Obtenido de https://developer.ibm.com/es/articles/que-es-big-data/

Cava, L. G. (2004). CRM: Tres estrategias de éxito. Barcelona: Gemma Tonijuan.

Citrix (02 de 09 de 2012). Revista Cloud Computing. El 95\% de las personas que utiliza servicios en la nube no sabe lo que es cloud computing. Obtenido de https://www.revistacloudcomputing.com/2012/09/el-95-de-las-personas-queutilizaservicios-en-la-nube-no-sabe-lo-que-es-cloud-computing/

Computerworld (2016). Impulsando la competitividad empresarial. Negocios \& Liderazgo Tic. No. 289, agosto 2016.

Cortés, F. (2012). Cómo Orientar el Potencial de Big Data. Obtenido de IBM Software Group, Information Management: ftp://public.dhe.ibm.com/software/es/events/doc/pdf/02_Fernando_Cortes_Co mo_Orie ntar_el_Potencial_de_Big_Data.pdf

Goyes Jiménez, K. M. (2017). Análisis de la actitud del consumidor respecto al uso del $\mathrm{M}$ - Commerce en las cadenas de autoservicio en el Distrito Metropolitano de Quito (DMQ). Quito: Universidad de las Fuerzas Armadas ESPE. Carrera de Ingeniería en Mercadotecnia.

López García, D. (2013). Análisis de las posibilidades de uso de Big Data en las organizaciones. Tesis de Maestría, Universidad de Cantabria.

Mayer-Schönberger, V. \&. (2013). Big data: la revolución de los datos masivos. España: Turner.

Proaño, M. (2011). Computación en nube. RETOS Revista de Ciencias de la Administración y Economía, 1.

Revista Líderes (17 de 09 de 2012). Las empresas ecuatorianas se proyectan a la nube. Obtenido de http://www.revistalideres.ec/lideres/empresas-ecuatorianasproyectan- nube.html

Uptime Institute (2017). Uptime Institute. Obtenido de https://es.uptimeinstitute.com/

Vallejo, J. (02 de 01 de 2017). El Ciudadano. Obtenido de http://www.elciudadano.gob.ec/ecuador-cuenta-con-mayor-velocidad-de- 


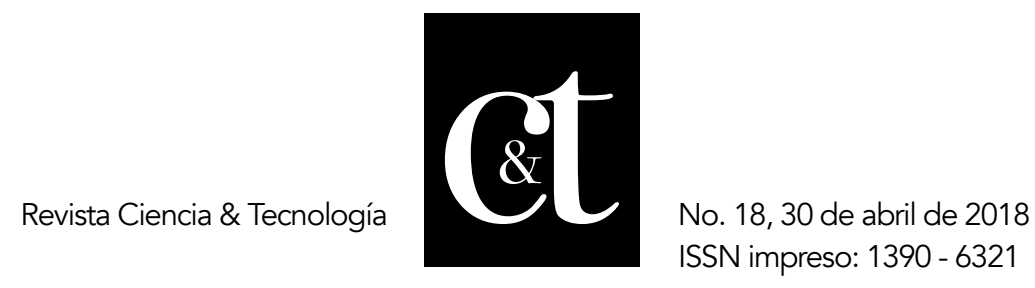

acceso- ainternet/

Viñals, J.T. (2012.a). Cloud Computing y Big Data, la próxima frontera de la innovación. FUNDACIÓN RAMÓN ARECES, 29-34.

Viñals, J.T. (2012.b). Del cloud computing al big data. Barcelona: Eureca Media. 